\title{
A Challenging Scenario in a Patient with A ST Elevation Acute Myocardial Infarction
}

\section{Introduction}

Treatment of bifurcated lesions in the setting of ST elevation acute myocardial infarction (STEMI) require high skills and ability by the interventional cardiologists. In the critical situation when the culprit lesion involves the distal segment of the left main coronary artery (LMCA) the choice of the best percutaneous revascularization technique is essential for patient outcome.

\section{Case Report}

We present a 45-year-old man that was admitted to an emergency department with a history of sudden-onset, severe central chest pain associated with nausea and dyspnoea. Regarding cardiovascular risk factors, he was an active smoker (20 daily cigarettes) and had dyslipidaemia. On hospital arrival, he was lucid and oriented, had normal heart sounds and fine right basal inspiratory lung rales; arterial blood pressure was $120 / 70 \mathrm{~mm} \mathrm{Hg}$ and heart rate 76 beats/min. The electrocardiogram showed sinus rhythm with ST segment elevation ( $4 \mathrm{~mm}$ in leads I and aVL, $2 \mathrm{~mm}$ in lead aVR), so emergent coronary angiography was indicated. The coronary angiogram showed an image suggestive of rupture plaque, that was located in the distal segment of the LMCA (Figure 1 ). There were also other lesions, a thrombotic total occlusion of the proximal circumflex $(\mathrm{CX})$ artery and a severe stenosis of the proximal left anterior descending (LAD) artery (Figures $2 \& 3$ ). Right coronary artery was dominant with no lesions.

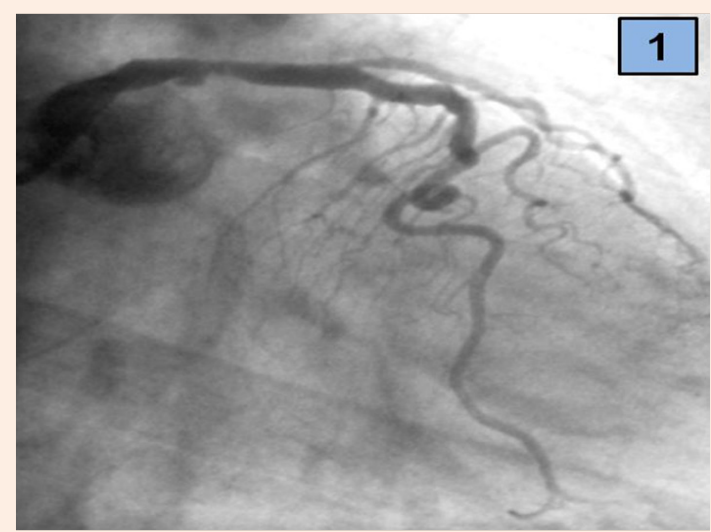

Figure 1: Severe distal LM and ostial LAD lesions with thrombotic occlusion of CX artery, AP view.

A seven French extra back-up catheter was placed in the ostium of the left coronary artery. Three guidewires were advanced: one in the LAD artery and the other two in the CX artery and branches. While crossing the CX occlusion with the wire, the vessel opened spontaneously (Figure 4) and an image suggestive of thrombotic material was seen in the $\mathrm{CX}$ and the second obtuse marginal branch (Figure 5).

Case Report
Volume 11 Issue 1 - 2018
Isaac Pascual, Alberto Alperi, Fico Pun-
Chinchay, Daniel Hernández-Vaquero, Rocío
Díaz, Rebeca Lorca, César Morís and Pablo
Avanzas*
Cardiac Catheterization Laboratories, Área del Corazón,
Hospital Universitario Central de Asturias, Spain
*Corresponding author: Pablo Avanzas, Cardiac
Catheterization Laboratories, Area del Corazón, Hospital
Universitario Central de Asturias, Oviedo, Spain,
Email: avanzas@secardiologia.es
Received: December 30, 2016| Published: January 08,
2018

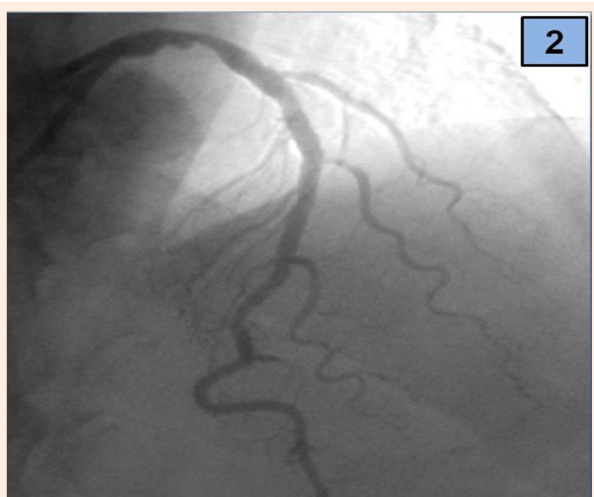

Figure 2: Severe distal LM and ostial LAD lesions with thrombotic occlusion of CX artery, cranial AP view.

Afterwards, $15 \mathrm{mg}$ of intracoronary abciximab were infused and a zotarolimus drug eluting stent (Resolute Integrity 3,5x22 $\mathrm{mm}$ ) was placed directly from the CX artery to the second obtuse marginal branch (Figures $6 \& 7$ ).

a) In order to treat the distal LMCA lesion, a CULOTTE STENTING technique was used:

b) A $3.5 \times 30 \mathrm{~mm}$ zotarolimus drug eluting stent (DES) was implanted between the LM artery and CX, overlapped to the previous stent (CX, Figure 8).

c) A guidewire was recrossed to LAD artery through the struts of the previously implanted stent (Figure 9). 
d) A $3.5 \times 18 \mathrm{~mm}$ zotarolimus DES was placed from medium LM to proximal LAD artery through the LM - CX stent (Figure 10 ), and a new guidewire was recrossed to the CX (Figure 11).

e) The procedure was successfully terminated (final TIMI 3 flow) by kissing balloon dilatation of both branches, with two non-compliant NC balloons of $3.5 \times 12 \mathrm{~mm}$ (Figure 12). Figures 12 and 13 show the final result.

The patient clinical status improved dramatically after PCI. He was discharged five days after admission.

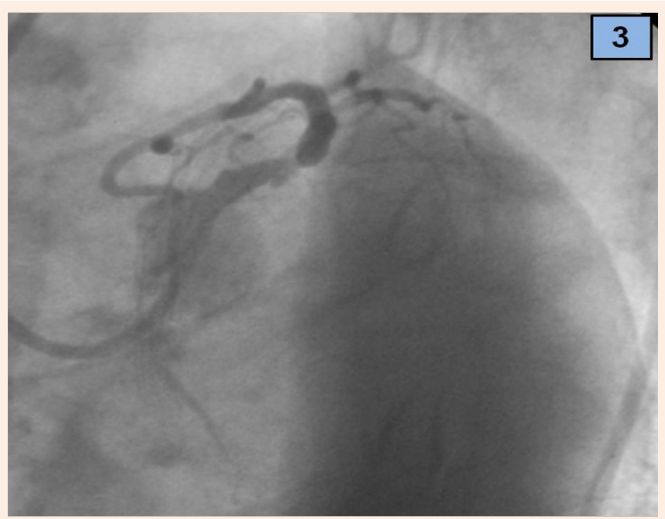

Figure 3: Severe distal LM and ostial LAD lesions with thrombotic occlusion of CX artery, LAO 50 Caudal 30 view.

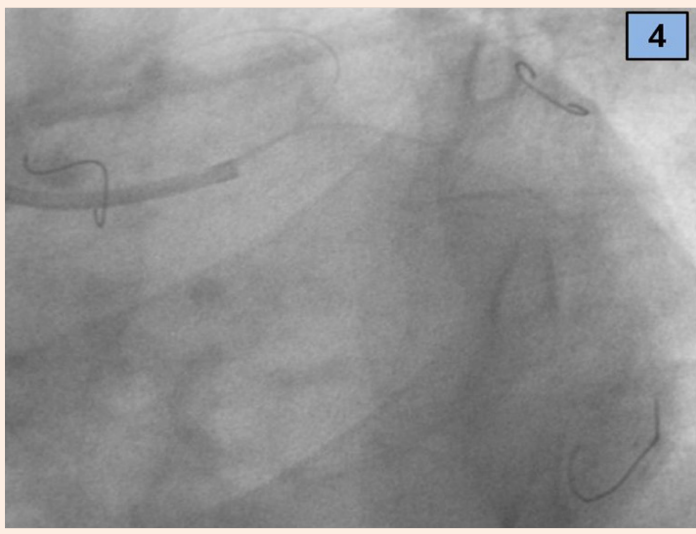

Figure 4: Three wires were placed, one in LAD and two in CX (marginal branches).

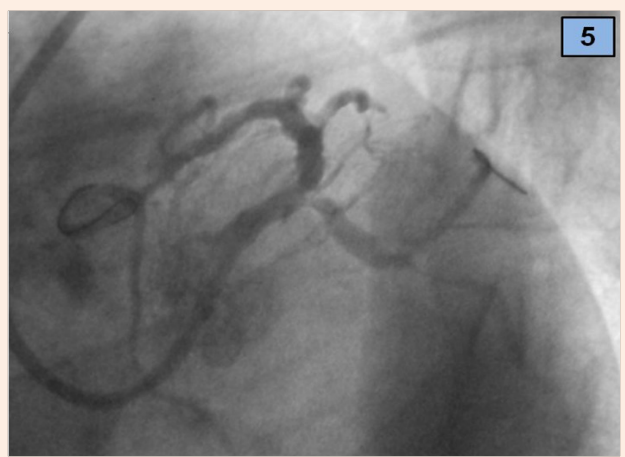

Figure 5: The CX artery opened spontaneously after crossing the lesion with the wire.

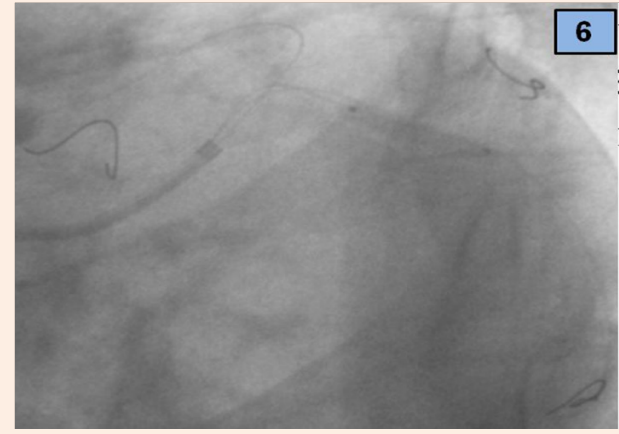

Figure 6: DES implantation (from CX artery to the second obtuse marginal branch).

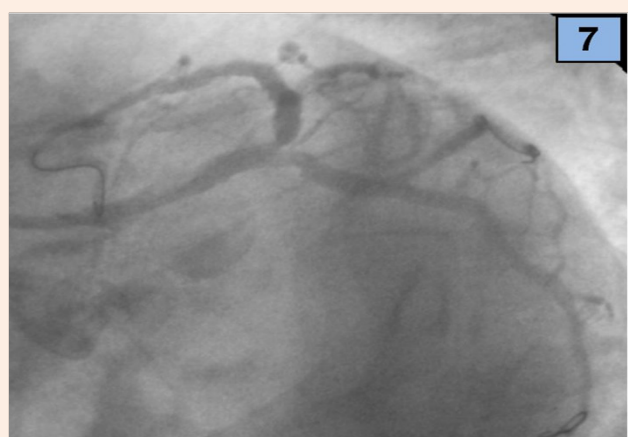

Figure 7: Result after DES implantation (CX artery to the second obtuse marginal branch).

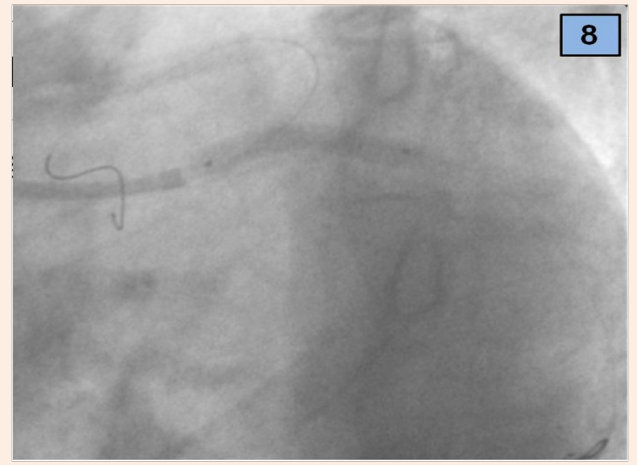

Figure 8: DES implantation between the LM artery and CX, overlapped with the previous one.

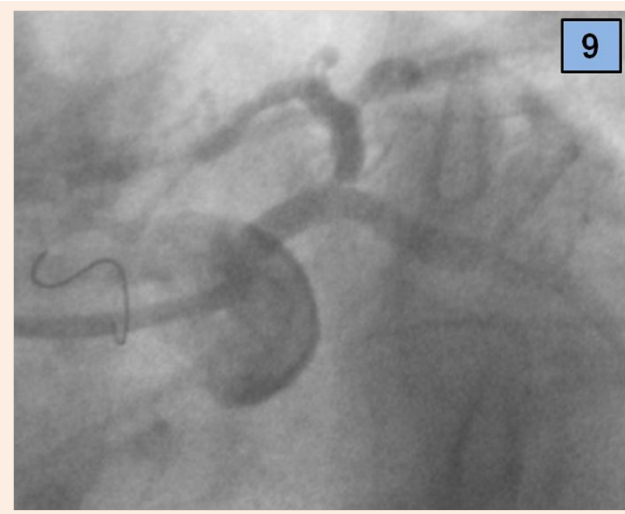

Figure 9: A wire was recrossed to LAD artery through the struts of the LM and CX stent. 


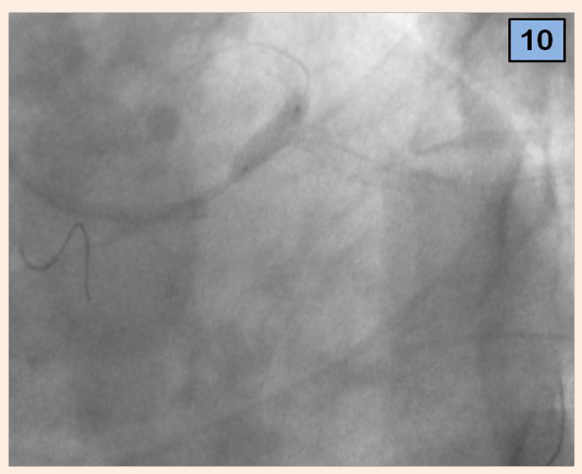

Figure 10: DES implantation (from medium LM to proximal LAD).

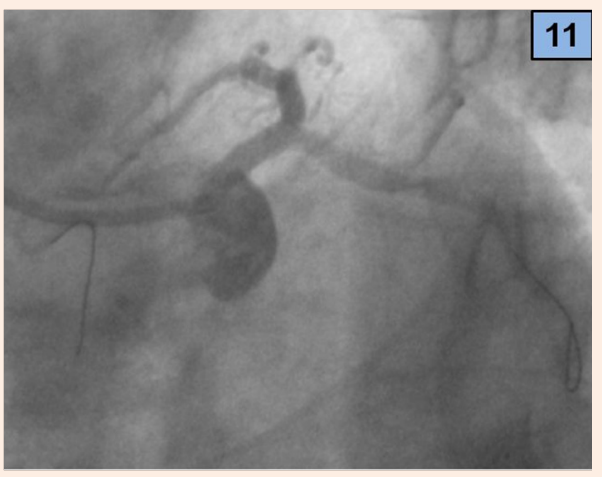

Figure 11: The wire in the CX was recrossed.

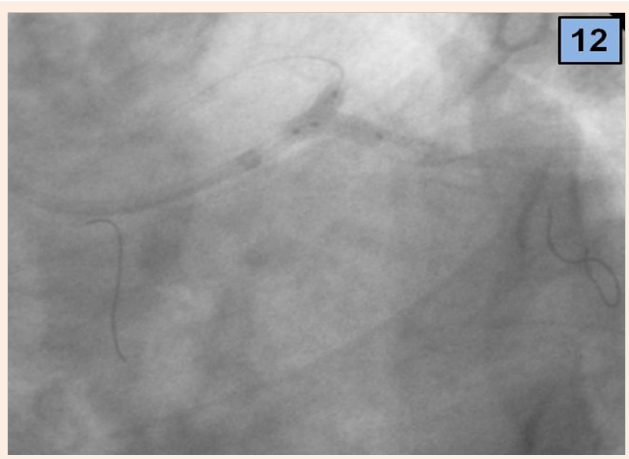

Figure 12: Final kissing balloon.

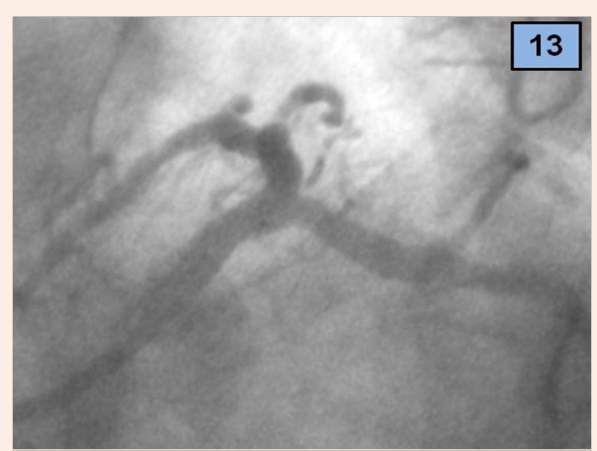

Figure 13: Final result, LAO caudal view.

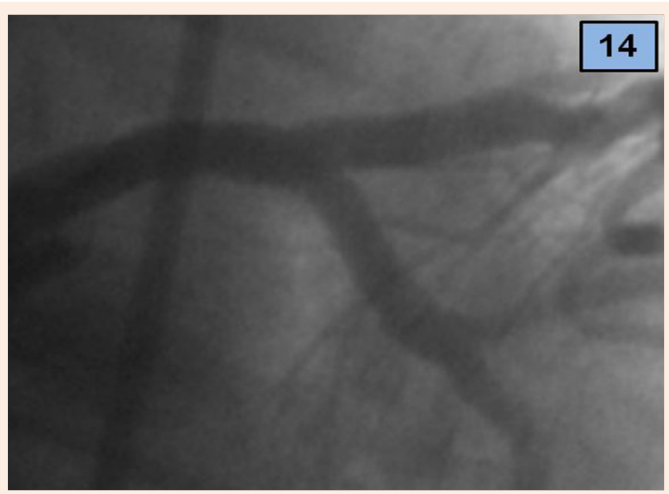

Figure 14: Final result, RAO view.

\section{Discussion}

Percutaneous interventions on lesions located on coronary bifurcations have been considered a challenging task for interventional cardiologists. When compared to non-bifurcation interventions, bifurcation interventions have a lower rate of procedural success, higher procedural costs, longer hospitalization and a higher rate of clinical and angiographic re-stenosis [1]. Before the DES era, the re-stenosis rate was unacceptably high on both branches regardless of the technique used. After DES became widely available, different studies evaluated the best procedural choice when percutaneous revascularization is needed for bifurcation lesions [2,3]. Results from different studies have shown that provisional stenting is as effective as any of the two stents techniques, so it should be the technique of choice in this setting.

But there is still controversy, because provisional stenting technique in some cases may cause shifting of the thrombus to the side branch, thus compromising its flow or even causing occlusion. On the other hand, when a two stents strategy is used to preserve the side branch, the amount of metal at the carina site and the presence of residual thrombus may cause early stent thrombosis. In our particular case, the patient presented with a STEMI. Because STEMI patients were excluded from most bifurcation trials, there are little data to support one technique for treatment over another. A retrospective study showed that in STEMI patients the culprit lesion involved a bifurcation lesion in $14 \%$ of the patients, the majority of them were LAD lesions (81\%). It evidenced no difference in the in-hospital adverse cardiovascular events between bifurcation and non-bifurcation patients [4]. A recent trial randomized symptomatic patients with large caliber true bifurcation lesions and significant ostial disease length to either a provisional T-stent strategy or a dual stent culotte technique. There was no difference between a provisional T-stent strategy and a systematic 2-stent culotte strategy in a composite end point of death, myocardial infarction and target vessel revascularization at 12 months. Nevertheless, STEMI patients were excluded in this study [5]. Results of the recently published BBK 2 trial showed that culotte stenting technique offer not only less per cent diameter stenosis but also less binary restenosis rate during follow up over the T-and-protrusion (TAP) 
stenting technique. There was also a consistent trend towards fewer target lesion re-interventions after culotte stenting as compared with TAP stenting [6].

In our case, due to the acute setting of the patient and the severe involvement of both branches, we think that using a culotte technique allows to cover all the coronary layers of the bifurcation lesion, avoiding any excessive "crushing" of displaced and distorted metal against the coronary wall. Performing an intravascular ultrasound imaging (IVUS) would have given better anatomical information, but due to the acute setting and clinical status of the patient, a prompt revascularization procedure was necessary.

In our opinion, the most suitable lesions for this approach are the Medina 0-1-1 and 1-1-1 bifurcation lesions, when there is significant involvement of the main branch, the diameter of the two vessels is quite similar and the angulation between them is suitable. The ability and experience of the operator in performing this technique is also crucial to obtain good results.

\section{References}

1. Renkin J, Wijns W, Hanet C, Michel X, Cosyns J, et al. (1991) Angioplasty of coronary bifurcation stenoses: immediate and longterm results of the protecting branch technique. Cathet Cardiovasc Diagn 22(3): 167-173.
2. Colombo A, Bramucci E, Sacca S, Violini R, Lettieri C, et al. (2009) Randomized study of the crush technique versus provisional sidebranch stenting in true coronary bifurcations: the CACTUS (Coronary Bifurcation: Application of the Crushing Technique Using SirolimusEluting Stents) Study. Circulation 119(1): 71-78.

3. Hildick-Smith D, de Belder AJ, Cooter N, Curzen NP, Clayton TC, et al. (2010) Randomized trial of simple versus complex drug-eluting stenting for bifurcation lesions: the British Bifurcation Coronary Study: old, new, and evolving strategies. Circulation 121(10): 12351243.

4. Kanei Y, Nakra NC, Liou M, Singh J, Fox JT, et al. (2013) The importance of bifurcation lesions in patients undergoing percutaneous coronary interventions in ST-segment elevation myocardial infarction. Cardiovasc Revasc Med 14(2): 81-83.

5. Hildick-Smith D, Behan MW, Lassen JF, Chieffo A, Lefèvre T, et al. (2016) The EBC TWO Study (European Bifurcation Coronary TWO): A Randomized Comparison of Provisional T-Stenting Versus a Systematic 2 Stent Culotte Strategy in Large Caliber True Bifurcations. Circ Cardiovasc Interv 9(9).

6. Ferenc M, Gick M, Comberg T, Rothe J, Valina C, et al. (2016) Culotte stenting vs. TAP stenting for treatment of de-novo coronary bifurcation lesions with the need for side-branch stenting: the Bifurcations Bad Krozingen (BBK) II angiographic trial. Eur Heart J 37(45): 3399-3405. 